\title{
Les capteurs radiométriques en sédimentologie dynamique
}

\author{
G. Meyer \\ Commissariat à l'énergie atomique
}

Prenant le relais de l'écho-sondeur ( 33 et $210 \mathrm{kHz}$ ), les capteurs radiométriques permettent une auscultation interne d'un massif sédimentaire (vase fluide). Cette exploration in situ présente des caractères intéressants pour un gestionnaire de barrage ou un responsable de l'entretien des chenaux d'accès portuaires:

- méthodes continues, sans perturbation du milieu mesuré ;

- méthodes globales, puisqu'elles ne nécessitent pas de prélèvements ;

- méthodes immédiates, les informations recueillies par les sondes apparaissent en clair: profondeur et concentration.

Suivant le type d'information souhaitée, on pourra utiliser, sur le terrain :

- des appareils "statiques " donnant suivant une verticale, le gradient de concentration;

- des appareils «dynamiques " permettant, par exemple, de repérer longitudinalement une ligne de densité choisie ; - des appareils à prélèvement continu, pour de faibles concentrations et/ou des conditions de mesures périlleuses pour l'appareillage.

En outre, il existe pour le laboratoire des instruments permettant :

- l'étude de carottes géologiques, du point de vue de la densité ;

- l'étude du tassement des sédiments en fonction du temps.

A tous ces capteurs sont associés une électronique de détection et de plus en plus fréquemment une saisie et un traitement informatique des informations.

\section{Principes des capteurs radiométriques}

En deux mots, nous pouvons dire qu'un capteur radiométrique est composé d'une source radioactive et d'un détecteur de rayonnements. Le choix du radioisotope en énergie et en activité dépend du type de problème à résoudre, de même que leurs dispositions relatives: on peut utiliser, soit l'absorption d'un faisceau de rayonnements, soit la diffusion de celui-ci. On optimisera lors de la conception de l'appareil, le choix entre la sensibilité et la précision de la mesure, suivant en ceci les options des demandeurs, qui peuvent privilégier l'une ou l'autre qualité, tout en laissant la possibilité de raffiner si le besoin s'en faisait sentir, une ou plusieurs mesures ponctuelles.

\section{Les appareils de terrain}

\subsection{Profils verticaux de concentration. La sonde JTD3}

L'une des applications classiques de cette sonde est la détermination de profils verticaux de densité dans les estuaires et les retenues de barrage. Ainsi que le montre la figure 1 , c'est une sonde à diffusion de photons. La source de césium 137 émet son rayonnement dans tout l'espace. Les photons émis en direction du détecteur sont absorbés par un écran de tungstène, si bien que seuls les photons diffusés par le milieu environnant peuvent atteindre le détecteur. La mesure in situ est précédée par un étalonnage, réalisé en général (mais ceci n'est pas obligatoire) avec les sédiments à mesurer.

\section{Radiometric sensors on dynamic sedimentology}

A radiometric sensor includes two main elements : a radioactive source and a radiation detector. The choices of the energy, the source activity, and the detector depend on the type of application to be carried out and of the expected accuracy. Two types of application are described using two different sensors. 

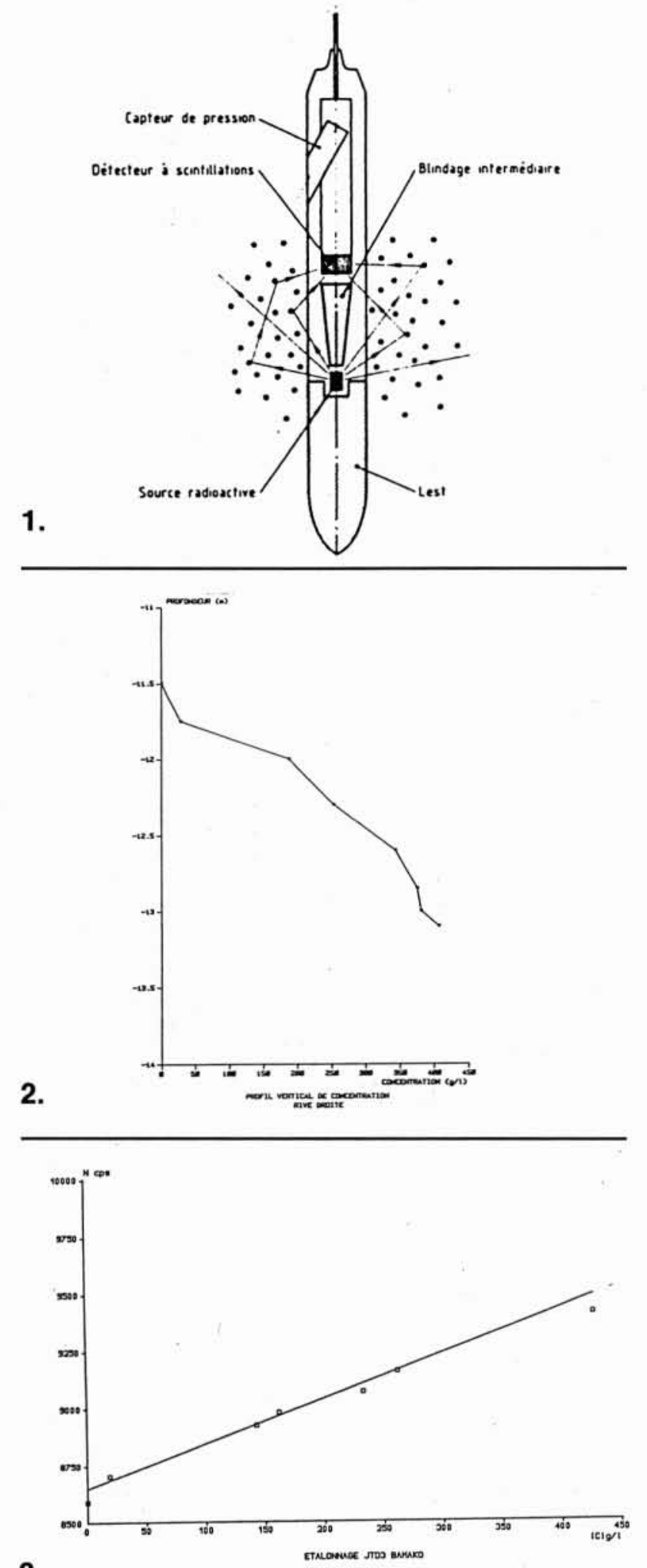

3.

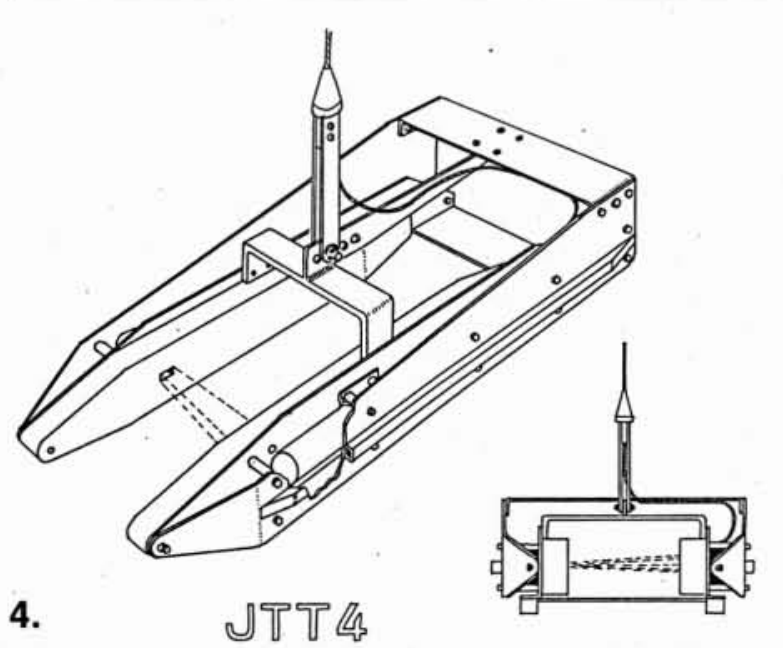

La figure 2 montre une telle courbe d'étalonnage réalisée à l'aide d'une suspension de kaolin, à l'Ecole nationale d'ingénieurs de Bamako (République du Mali).

Après la réalisation de l'étalonnage de la partie nucléaire qui donnera la valeur de la concentration et celui du capteur de pression qui déterminera la profondeur de la zone mesurée, l'ensemble de l'appareil: sonde, treuil, micro-calculateur (Apple ou compatible PC) est placé à bord d'une embarcation qui peut être de dimensions réduites, compte tenu du faible encombrement de l'ensemble. Là, les mesures sont réalisées à raison de 80 profils par jour (voir l'exemple de la figure 3), ce qui permet de couvrir une surface d'estuaire non négligeable dans le but de réaliser par exemple une cubature avant et après une campagne de dragage, ou bien de suivre en fonction du temps la sédimentation dans une retenue de barrage et d'aider ainsi à la gestion de l'ouvrage.

La verticalité de la sonde pendant la mesure est l'un des critères essentiels: le volume de mesure, c'est-à-dire le volume de matériau dans lequel se produit l'interaction rayonnement-matière avec une probabilité non nulle pour que le photon Compton revienne au détecteur, a approximativement la forme d'un cylindre plat de $700 \mathrm{~mm}$ de diamètre et de $100 \mathrm{~mm}$ de hauteur, son axe étant confondu avec celui de la sonde. Si celle-ci est inclinée, le volume de mesure va intéresser vers le haut des couches de faible densité et vers le bas de couches de forte densité. La mesure est donc faussée parce que moyennée autour d'une valeur qui peut être très éloignée de la valeur vraie.

Il est donc nécessaire de travailler avec des vitesses de courant d'eau inférieures à $1 \mathrm{~m} . \mathrm{s}^{-1}$. Pour les retenues de barrage cette condition est remplie la plupart du temps; pour les estuaires soumis à marée, il est clair que l'on ne peut travailler qu'aux étales de pleine ou basse mer, dans les heures de renverse. C'est la limitation principale de la sonde JTD3. La seconde barrière étant la limite de "pénétrabilité » de la sonde, dans un massif consolidé.

La gamme de mesure est comprise en 50 et $800 \mathrm{~g} \cdot 1^{-1}$.

L'examen des profils permet aussi de tracer des lignes d'isodensité ; mais pour ce genre d'expérimentation, on préférera utiliser, si la géographie du site le permet, une sonde tractée décrite dans le paragraphe suivant.

\subsection{Courbes d'isodensité. La sonde JTT4}

Cette sonde en est encore au stade de l'expérimentation sur le « terrain ». Le Port Autonome de Nantes-Saint-Nazaire qui, il y a quelques années, avait mis à notre disposition les moyens à la mer pour la mise au point in situ de la sonde JTD3, a bien voulu recommencer l'expérience avec JTT4.

D'abord définie par le calcul, puis expérimentée du point de vue du comportement hydrodynamique dans les bassins de l'Ifremer à Brest, la sonde JTT4 est actuellement entre les mains d'un personnel déjà aguerri aux techniques nucléaires en sédimentologie.

Cette expérimentation est primordiale, car elle met en relief les défauts imprévisibles de cet appareil d'un type nouveau, et elle permet aussi d'en apprécier les qualités et d'entr'apercevoir les utilisations et les résultats futurs. 
L'emploi de ce type de sonde, tractée par un bateau dans le massif sédimentaire d'un estuaire, permet d'aller repérer les profondeurs correspondant à une ou deux densités préalablement choisies (fig. 5) ou d'explorer entre deux profondeurs prédéterminées, les différentes couches à densité variable (fig. 6).

Ces lignes d'isodensité permettront de caler le «zéro hydro " des cartes à une valeur correspondant non plus à l'indication de l'écho-sondeur (profondeur de toit de vase), mais à une valeur correspondant à la limite de navigabilité. Ceci laisse entrevoir des économies substantielles, en travail et partant en coût, sur les frais de dragage des chenaux de navigation. Le taux d'utilisation temporel de la sonde JTT4, est supérieur à celui de JTD3 puisque l'influence de courants de marée est considérablement atténuée : il n'est plus nécessaire d'attendre les étales de basse mer ou de pleine mer pour utiliser l'appareil.

\subsection{Mesures par prélèvements continus}

Ces mesures sont réalisées lorsque le risque de destruction de l'appareillage s'accroît : en particulier dans les oueds en crue où sont charriés, outre les sédiments à mesurer, des matériaux de natures diverses : arbres, animaux emportés par la crue, etc... Dans ces conditions, il est jugé plus expédient de pomper dans la rivière, de façon continue et à des hauteurs variables la suspension eau-sédiment et d'en mesurer, par absorption de rayonnement, la concentration en matériau solide, à l'aide d'une cellule située sur un pont ou sur la rive. On se met ainsi à l'abri de la perte d'une source radio-active qui bien que de faible activité impressionne toujours, et à juste titre, un public parfois insuffisamment informé.

Cette méthode permet une exploration dans les trois dimensions du lit d'une rivière en crue et d'en déduire la quantité de sédiments transportée. Elle peut être utilisée aussi lorsque l'on ne peut introduire directement JTD3 et JTT4 sur le lieu où l'on souhaite effectuer la mesure. Des expérimentations ont été faites sur les canalisations de rejet des eaux d'une centrale nucléaire, pour mesurer le turbidité des eaux en sortie d'échangeur, les concentrations étant trop faibles pour pouvoir utiliser les jauges classiques sur canalisation.

\section{Appareils de laboratoire}

Ils concernent deux types de problèmes.

a) Examen des variations de densité le long d'une carotte sédimentaire; celle-ci pouvant être de nature rocheuse ou de nature sédimentaire consolidée. Ainsi que le montre la figure 7 , on peut distinguer (après un étalonnage préliminaire) différents types de sédiments, des fractures, des inclusions, etc... Il faut signaler aussi la possibilité d'associer à cette mesure, une mesure complémentaire de susceptibilité magnétique. Ce banc horizontal de gamma densimétrie permet l'examen de carottes ayant une

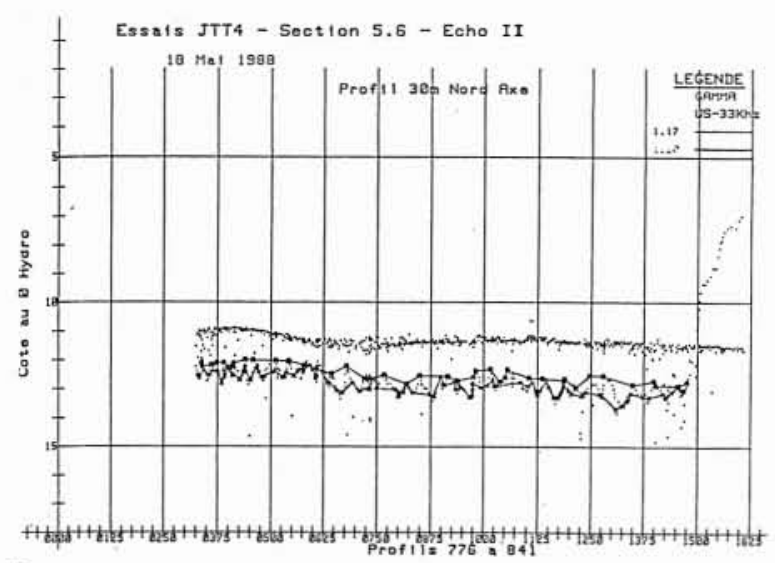

5.

6.

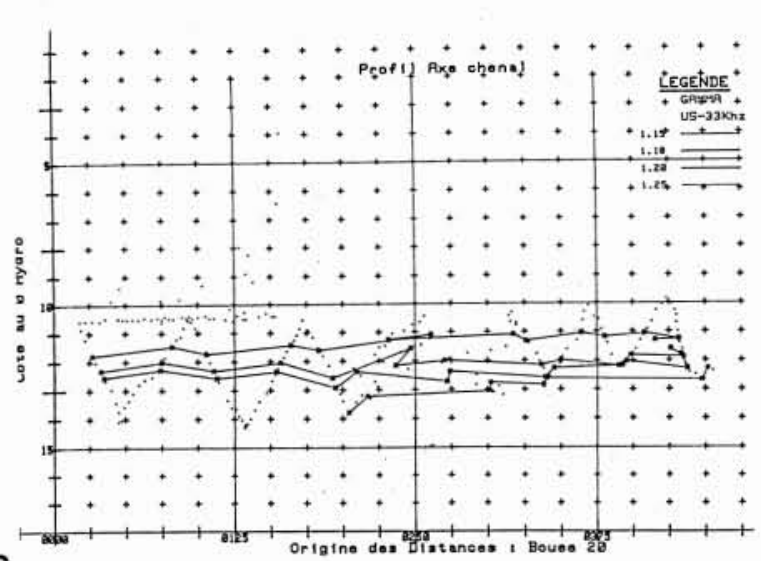

1. Principe de la jauge à diffusion JTD3.

2. Courbe d'étalonnage de JTD3.

3. Profil vertical de densité (Barrage d'Inga, République du Zaïre).

4. Schémas de principe de la jauge à transmission JTT4.

5. Profil longitudinal: recherche de 2 densités.

6. Profil longitudinal: exploration d'un massif sédimentaire.

7. Examen gamma densitométrique d'une carotte sédimentaire. 
longueur maximale de 3 mètres. Par un entraînement en rotation de l'échantillon, une exploration hélicoïdale de l'échantillon est réalisable.

b) Etude du tassement de sédiments

On dispose de deux types d'appareillages, différant l'un de l'autre par leurs dimensions: une jauge permettant l'exploration verticale et l'évolution d'une suspension homogène sur une hauteur de $1 \mathrm{~m}$ environ l'autre sur une hauteur de $3 \mathrm{~m}$. Mais le principe de ces jauges est le même : un équipage mobile se déplace le long de l'échantillon et mesure en chaque point l'absorption d'un rayonnement par le matériau contenu dans l'éprouvette.

La figure 8 montre un type d'évolution sur 60 jours de deux suspensions initialement homogènes, pour deux concentrations différentes à l'origine.

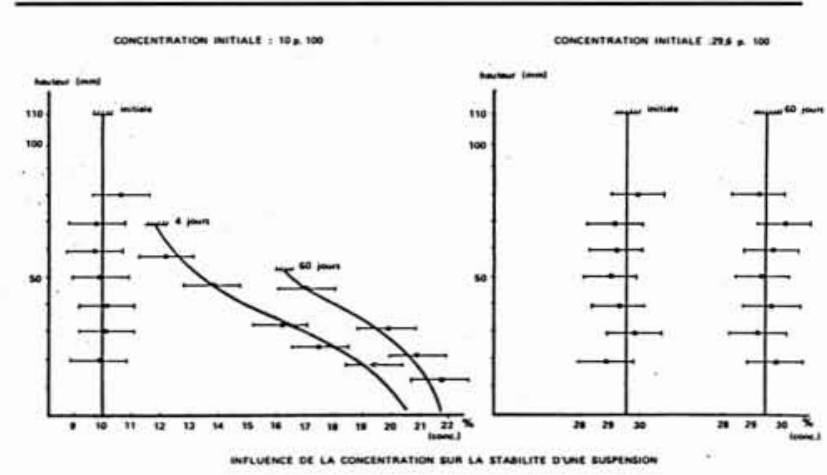

8. Etude de la décantation d'une suspension en fonction du temps. .

\section{Conclusions}

Les appareils que nous avons succinctement décrits et dont nous avons montré quelques possibilités répondent à des demandes d'exploitants de ports ou de gestionnaires de grands barrages.

L'atout majeur, semble-t-il, de ce type d'instrument réside dans le caractère global de l'information fournie. Le problème de la représentativité du prélèvement ne se pose pas puisque la mesure est réalisée in situ: de façon dynamique avec JTT4, de façon plus ponctuelle avec JTD3, et de manière différente mais continue quand les impératifs de sécurité l'exigent.

L'instrumentation nucléaire n'est qu'un des volets des utilisations possibles de la radio-activité en milieu marin et portuaire : les traceurs radio-actifs permettent de suivre des déplacements de sédiments : érosion des littoraux, rejets de produits de dragage, etc...

Quant aux instruments de laboratoires, ils fournissent des données prévisionnelles ou des contrôles a posteriori et répondent plus aux besoins des chercheurs qui forment les équipes indispensables aux exploitants. 